\title{
Two-Dimensional Chiral Molecular Networks from Achiral Building Blocks: A Computational Study
}

\author{
Paweł Szabelski • Adam Kasperski
}

Published online: 18 October 2011

(c) The Author(s) 2011. This article is published with open access at Springerlink.com

\begin{abstract}
This article describes the application of computer simulations to explore the self-assembly of model achiral molecules on a solid surface leading to the creation of chiral overlayers. To that purpose the lattice gas Monte Carlo method is used to trace the spontaneous self-organization of cross- and tripod-shaped molecules which are represented by rigid planar structures comprising interconnected segments. The study focuses mainly on the influence of size and composition of the molecules on the morphology of the resulting superstructures. It is clearly demonstrated that the molecules, although intrinsically achiral, can assembly into globally chiral two-dimensional networks with regular cavities. Our simulations show also how the chiral networks can be obtained via co-assembly with much smaller molecules and how the additive fills the cavities. In this case, the mixed superstructure is further used as a model enantioselective adsorbent whose adsorptive properties are examined by simulating adsorption isotherms of a racemic mixture of a prototype chiral molecule. The results of this part indicate that the achiral molecular building blocks can be used to construct enantioselective surfaces with tunable adsorption properties.
\end{abstract}

Keywords Self-assembly · Monte Carlo simulation · Chirality $\cdot$ Adsorption $\cdot$ Pattern formation

P. Szabelski $(\bowtie) \cdot$ A. Kasperski

Department of Theoretical Chemistry, Maria-Curie Skłodowska

University, Pl. M.C. Skłodowskiej 3, 20-031 Lublin, Poland

e-mail: szabla@vega.umcs.lublin.pl

\section{Introduction}

Imparting chirality to solid surfaces has been long a challenging task in the fabrication of enantioselective adsorbents and catalysts [1]. Preferential adsorption of one enantiomer over the other is a basic requirement for effective chiral adsorptive separations and stereoselective heterogeneous catalysis. Usually, a chiral surface comprises chiral centers or selectors which interact more strongly with the preferred enantiomer, so that it can be selectively captured from a racemate. This property has been extensively used in chiral chromatography, in which stationary phases are often built of small chiral organic molecules attached to a silica surface [2]. In those enantioselective adsorbents called Pirkle phases the linked organic molecules play the role of chiral selectors providing differentiation between enantiomers of chiral alcohols, aminoacids, ketones, esters etc. [2, 3].

Recently there has been a growing interest in alternative methods of fabrication of chiral surfaces. Those methods are based on the use of single metallic crystal faces which are either naturally chiral [4] or they are modified by adsorption of organic molecules [4-6]. Naturally chiral surfaces are usually obtained by cleaving of achiral bulk metallic structures $[7,8]$ or exposing of surfaces of naturally chiral crystals $[9,10]$. The resulting surface has a unique structure, for example a unique arrangement of kinks and steps, which is not superimposable with its mirror image and thus it is chiral. Because of the chirality, the surface interacts more strongly with those molecules which are complementary to the surface, that is, they fit chiral nanostructures chiraly. In the case of chiraly templated surfaces, a metallic crystal face is covered by small chiral template molecules such as tartaric acid [11], cinchona alkaloid [4], or 2-butanol [12,13] which self-organize to form chiral cavities which are believed to be 
the source of preferential adsorption of chiral guest molecules such as propylene oxide $[6,13]$ or 2-aminobutanoate [14].

The concept of creating ordered void spaces on solid surfaces to achieve preferential adsorption or deposition of a certain molecule has been particularly important not only to adsorption and catalysis but also to nanoengineering and material chemistry [15]. For example, the cavities apart from being environment for adsorption or for catalytic reactions in confined space, can be filled with matter of predefined optical, magnetic or biological activity. However, a challenging task is to develop methods which allow for the fabrication of extended porous structures in a fully controlled and repetitive way. A promising method in this field seems to be the self-assembly of functional organic molecules on metals or graphite either under vacuum conditions [15-17] or from liquid phase [18, 19]. As it has been demonstrated for numerous systems, suitably chosen molecular building blocks can assembly into extended twodimensional porous networks with cavities whose shape and size can be finely tuned by manipulating properties of the building block [15-19]. Moreover, it has been shown that even achiral molecules can form globally chiral ordered structures when assembled in one-component systems [20, 21] or co-assembled with elementary metals [22]. Those networks are usually obtained using molecules which form intermolecular hydrogen bonds or coordination bonds with additive metal atoms. It has been also demonstrated that the extended chiral porous networks can form spontaneously via van der Waals interactions, by interdigitation of long alkyl chain arms of the adsorbed star molecules [18, 23].

A quite surprising observation form the experimental studies is that in some instances the shape of a building block, not its chemical composition, directs the morphology of the resulting structure. For example, it can be observed that three chemically different molecules of a tripod shape, including annulenes derivatives and polyaromatic rigid tripods ensemble on graphite into isomorphic chiral structures with hexagonal pores [23-25]. This observation suggests that the structure of a selfassembled layer can be sometimes predicted, based on the shape and size of the building block. Inspired by this idea, in this work we propose a theoretical model of the selfassembly of achiral star molecules into two-dimensional chiral porous networks. To that purpose we consider tripod- and cross-shaped molecules, with a special focus on the latter, as the superstructures formed by these building blocks are further demonstrated to be chiral medium for adsorptive separation of chiral guest molecules.

\section{The Model and Simulation of Self-Assembly}

In order to model the self-assembly in adsorbed layers we used two types of planar molecular structures which are shown schematically in Fig. 1. The molecules from Fig. 1 were assumed to be rigid and composed of a central segment called core connected with 3 (left) or 4 (right) $n$-membered arms. Depending on symmetry elements, the molecules are further called tripod-shaped $\left(C_{3}\right.$ axis) and cross-shaped ( $C_{4}$ axis), as indicated in the figure. These two structures were inspired by the shape of molecules such as derivatized annulenes [18, 23] and metal phthalocyanines [26-28], respectively. To mimic the formation of intermolecular donor-acceptor bonds the molecular arms were terminated with segments whose energetic parameters can differ from the rest of the molecule. We consider two qualitatively different situations in which (1) the molecules are equipped with active terminal segments and (2) they are composed of identical segments. The molecules belonging to the first and second group are called heterogeneous and homogeneous, respectively.

The tripod- and cross-shaped molecules were placed on a triangular and square lattice, respectively. In both cases
Fig. 1 Model structures used in the simulations: left the tripodshaped molecule adsorbed on a triangular lattice and right the cross-shaped molecule adsorbed on a square lattice. Each molecule comprises a central segment called core and 3 or 4 $n$-membered arms attached to it (molecules with $n=3$ are shown as an example). The terminal segments are encircled in red. The co-adsorbing additive molecule in shown in green
TRIPOD

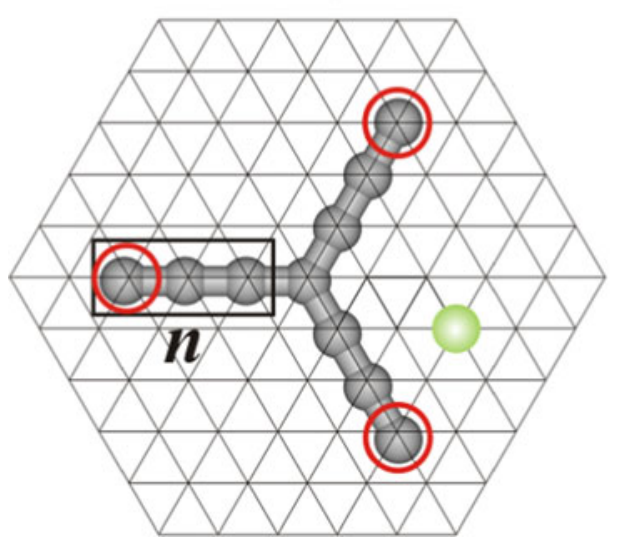

CROSS

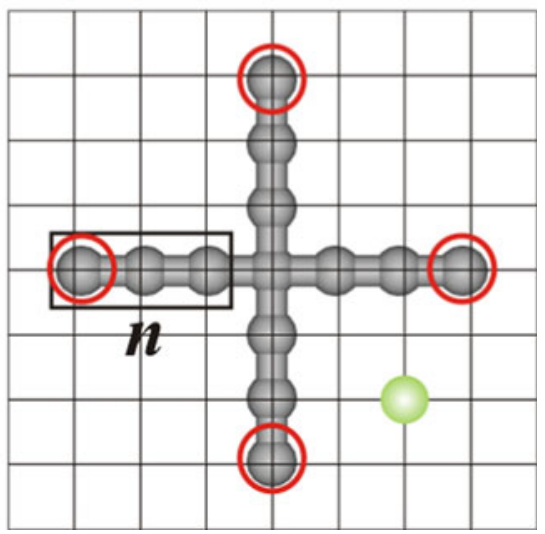


the lattice spacing $l=1$ was assumed to be equal to the intramolecular segment-segment distance, so that each segment occupied one lattice site. It was assumed that the modeled surface is energetically homogeneous. For the sake of convenience the molecule-surface interaction energy was set to 0 , as this energy does not affect the probability of moving a molecule to a new position on the homogeneous lattice. The molecules were assumed to interact via a short-ranged segment-segment interaction potential limited to nearest-neighbors on the corresponding lattice. The energy of interaction between a terminal segment of one molecule $(t)$ and a non-terminal segment $(n)$ of a neighboring molecule (including the core) was characterized by $\varepsilon_{n t}$. For like segments the energy of interaction was described by $\varepsilon_{t t}$ for a pair of terminal segments and by $\varepsilon_{n n}$ for a pair of non-terminal segments. In the case of homogeneous molecules the segment-segment interaction energy was characterized by a single parameter $\varepsilon=\varepsilon_{n n}=\varepsilon_{n t}=\varepsilon_{t t}$. These energies are expressed in $k T$ units. According to the convention adopted here positive values correspond to intermolecular attraction.

To model the co-assembly of tripod- and cross-shaped molecules with atoms or with much smaller compact molecules we used a simplified approach in which these additives were represented by a single segment occupying one lattice site, as shown in green in Fig. 1. The energy of interaction between the additive and a terminal segment of a tripod- or cross-shaped molecule was characterized by $\varepsilon_{a t}$ while that between the additive and a non-terminal segment by $\varepsilon_{a n}$. Interactions between molecules of the additive were neglected.

For the tripod-shaped molecules the simulations were performed on a $L \times L$ rhombic fragment of a triangular lattice whereas for the cross-shaped molecules the underlying lattice was of a square $L \times L$ shape. In both cases we used the conventional canonical ensemble Monte Carlo (MC) method with Metropolis sampling. A detailed description of the simulation algorithm can be found in our previous work [29]. To eliminate edge effects periodic boundary conditions in both directions were applied to the considered lattices. To equilibrate the system we used up to $10^{7} \times N$ MC steps, where $N$ is the total number of molecules on the lattice and one $\mathrm{MC}$ step is defined as a single attempt to rotate and move a molecule to a new position. In most of calculations described here $N$ varied between 500 and 4500 . We note that the results obtained in this work hold for low temperatures at which the energy of the segment-segment interaction determines the extended structures and entropy effects are negligible.

\section{Results and Discussion}

\subsection{Self-Assembly in One-Component Systems}

To compare equilibrium superstructures formed by tripodand cross-shaped molecules with arms of a different length we first performed simulations for relatively small systems comprising 200 molecules adsorbed on lattices with $L=100$. The results obtained for the homogeneous tripodshaped molecules, for $\varepsilon=1$ and $n$ changing from 1 to 4 are shown in Fig. 2.

This figure presents small fragments of highly ordered domains which were observed and analyzed in detail in our previous work [29]. Here we comment briefly on their structural properties and compare them with analogous results obtained for the cross-shaped molecules. As it can be seen in Fig. 2, the tripod-shaped molecules with $n=1$ assemble into a closely packed structure which can be locally described by a simple $(2 \times 2)$ unit cell [30]. Because of the compact shape these molecules can form an
Fig. 2 Small fragments of the ordered overlayers formed by the homogeneous

$\left(\varepsilon_{t t}=\varepsilon_{n t}=\varepsilon_{n n}=1\right)$ tripod molecules with arm length equal to $1,2,3$ and 4 . The thick red line shows the rhombic unit cell of each structure. The black arrows in the bottom-right panel indicate the reference lattice axes. The side, $a$ and rotation angle, $\gamma$ of the unit cell are also defined in this panel. The black circular arrow shows the rotation direction of the pore

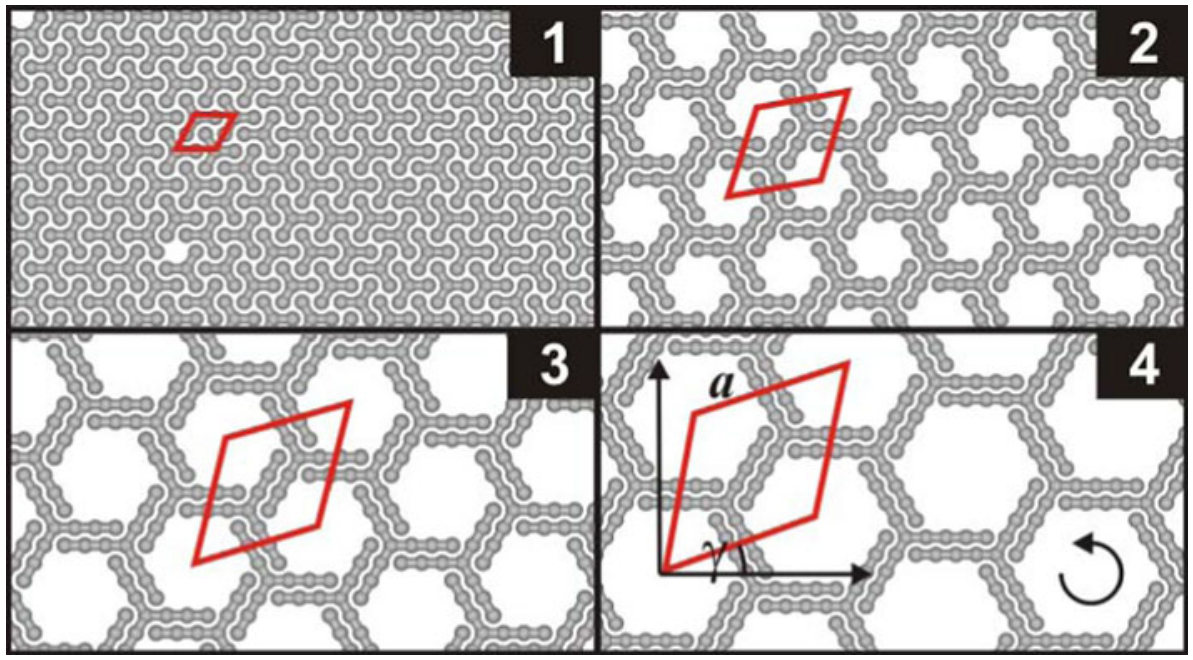


overlayer being a mixture of stripe domains in which they are oppositely oriented. In this case energetic properties of the molecules at the domain boundaries and inside the domains are identical which leads to the formation of a compact pattern.

A much more interesting situation is encountered when the arm length of the tripod-shaped molecules exceeds 1 . In this case, the molecules with $n>1$ form a porous pattern with hexagonal cavities which is characterized by a rhombic unit cell. In this pattern half of the molecules are rotated by $60^{\circ}$ with respect to the other ones, contrary to the pattern simulated for $n=1$ for which all molecules have the same orientation within one domain. The structures obtained for $n>1$ are isomorphic and the parameters characterizing their rhombic unit cell, including the side, $a$ and rotation angle, $\gamma$ increase noticeably with the number of segments in the arm. A distinctive feature of the molecular networks obtained for $n>1$ is that they are homochiral. As we observed [29], equilibration of the system, also at higher packing densities, leads to the formation of one large domain of a single handedness. This property is a direct consequence of the energy penalty associated with formation of boundaries between domains of the opposite handedness. An explanation of this effect relies on the fact that the molecules in our model are rigid and they cannot bent or partially desorb their arms. In real situations this effect can largely stabilize mirror-image domains which are in contact [23]. For that reason, the model tripod-shaped molecules assembly into one extended cluster whose net handedness is a result of a random choice. Occurrence of a right- and lefthanded domain at equilibrium is equally probable in the simulations. We note that, despite this drawback, the patterns shown in Fig. 2 reproduce morphology of the experimental self-assembled domains obtained using various chemically different tripod-shaped molecules [23-25].
An important note about the structures formed by the tripod molecules has to be made here. Namely, in the simulations described in this work we used low adsorbate densities which guarantee the formation of the porous hexagonal networks. However, it can be easily shown that at sufficiently high adsorbate coverages the tripod molecules assembly into a closely packed adlayer in which all they have the same orientation. Another reason for using the low surface coverage was to eliminate problems with periodic boundary conditions when the periodicity does not match the translational symmetry of the adlayer structure. This refers also to the crossshaped molecules.

Regarding the cross-shaped molecules, we performed analogous simulations in which the parameters were the same as in the case of the tripod-shaped molecules, that is: $L=100, N=200$ and $\varepsilon=1$. The results obtained for the homogeneous molecules with $n=1,2,3$ and 4 are shown in Fig. 3. As it can be seen in the figure, the cross-shaped molecules form highly ordered patterns, regardless of the arm length. Note that, contrary to the tripod molecules with $n=1$, the cross-shaped molecules assembly into a highly ordered compact $(\sqrt{5} \times \sqrt{5})$ structure resembling those observed experimentally for phthalocyanines [27]. In the remaining cases the resulting networks comprise square voids of increasing area. A common feature of the patterns shown in Fig. 3 is the square unit cell whose side and rotation angle increase with the elongation of the arm, as already observed for the tripod-shape molecules. Moreover, as in the previous case, the obtained patterns (including that one with $n=1$ ) are all homochiral. The reason for this is again the molecular rigidity and the aforementioned energy penalty associated with the formation of boundaries between domains of the opposite rotation.
Fig. 3 Small fragments of the ordered overlayers formed by the homogeneous $\left(\varepsilon_{t t}=\varepsilon_{n t}=\varepsilon_{n n}=1\right)$ crossshaped molecules with arm length equal to 1, 2, 3 and 4 . The thick red line shows the square unit cell of each structure. The meaning of the symbols and arrows is the same as in Fig. 2

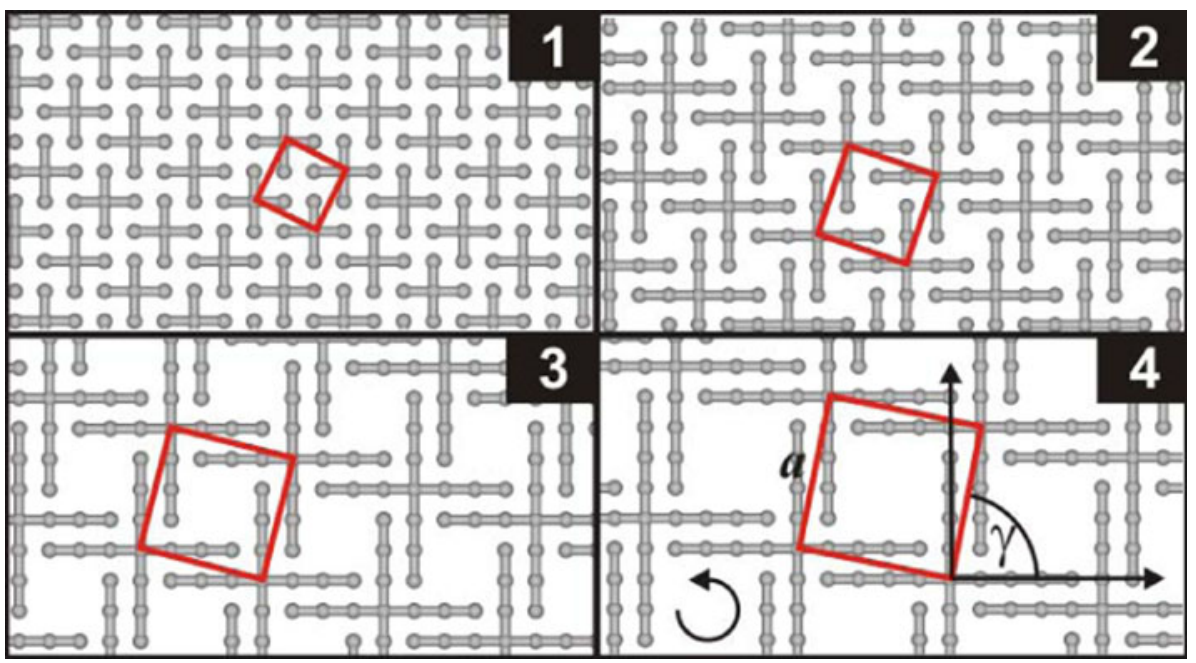


To study the effect of chemical composition of the molecules on the structure of assemblies they form we performed simulations in which the terminal segments were activated. To that purpose we assumed that $\varepsilon_{t t}=2$ and $\varepsilon_{n t}=\varepsilon_{n n}=1, L=100$, and $N=200$. The results simulated for the tripod-shaped molecules are presented in Fig. 4 which shows small fragments of the porous networks comprising molecules with $n=2$ and 3 .

As it follows from Fig. 4, activation of the terminal segments influences considerably morphology of the networks formed by the tripod-shaped molecules with $n>1$. In the case of the shorter arm $(n=2)$, the homochiral porous structure from Fig. 2 converts into achiral linear pattern when $\varepsilon_{t t}$ grows from 1 to 2 . This structure is characterized by a rectangular unit cell and contains short linear voids ( 2 adsorption sites in length). Even more profound changes can be observed in the case of $n=3$. Here, the pattern shown in the right part of Fig. 4 is homochiral but the network comprises pores of two types: bigger rhombic and smaller parallelogram. The whole structure is then characterized by a parallelogram unit cell. The results of the simulations performed for the tripodshaped molecules demonstrate that, in general, manipulation of their size and chemical composition leads to the formation of largely diversified patterns which do not simply scale with $n$. This in contrast with what was observed for the homogenous molecules with $n>1$.

In the case of the cross-shaped molecules, activation of the terminal segments is responsible for the formation of scalable porous networks with rectangular cavities. Examples of the networks simulated for $n=2$ and 4 are shown in Fig. 5. These structures are small fragments of large ordered domains obtained for $\varepsilon_{t t}=2$ and $\varepsilon_{n t}=\varepsilon_{n n}=1, L=100$, and $N=200$. The surface patterns presented in Fig. 5 are homochiral and they are characterized by a square unit cell, as shown in red. The homochirality of these simulated patterns has been explained before.

To summarize and compare quantitatively the results obtained so far, in Table 1, we listed main structural parameters of the scalable porous networks. These parameters are expressed as functions of the arm length and they were derived for an arbitrary $n$. Figure 7 shows the pore area (left) and saturation capacity, $C$ (right) plotted using the expressions from Table 1 . The saturation capacity was defined as the number of unoccupied lattice sites within the unit cell divided by the area of that cell. Both these quantities are relevant to adsorption properties of the self-assembled structures whose pores can accommodate guest molecules. The first one characterizes the maximum size of a guest molecule which can fit a cavity while the latter informs about the total space available to small molecules, for example such that each of them occupies one lattice site. As it clearly follows from Fig. 6, the pore area increases most rapidly with $n$ when the tripod-shaped molecules are used. For example, the pore area calculated for tripod-shaped molecules with $n=4$ is about three times larger than analogous results obtained for the remaining building blocks. Note also that the corresponding plots calculated for the cross-shaped molecules are very similar in shape (see red and grey lines). However, those molecules form slightly larger pores when equipped with

Fig. 4 Small fragments of the ordered overlayers formed by the heterogeneous $\left(\varepsilon_{t t}=2\right.$ and $\left.\varepsilon_{n t}=\varepsilon_{n n}=1\right)$ tripod molecules with arm length equal to 2 and 3 . The thick red line shows the corresponding rectangular $(n=2)$ and parallelogram $(n=3)$ unit cells
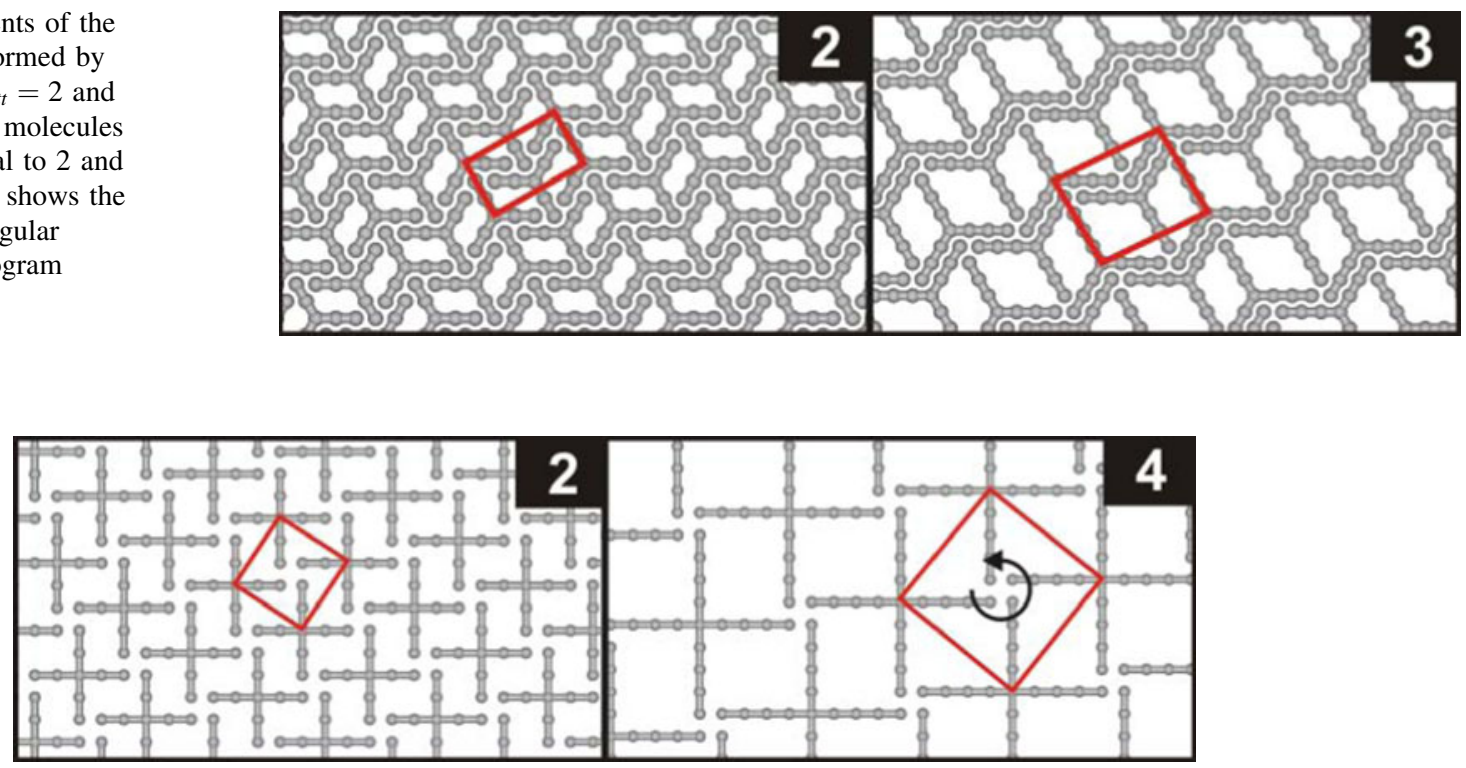

Fig. 5 Small fragments of the ordered overlayers formed by the heterogeneous $\left(\varepsilon_{t t}=2\right.$ and $\left.\varepsilon_{n t}=\varepsilon_{n n}=1\right)$ cross-shaped molecules with arm length equal to 2 and 4 . The thick red line shows the square unit cell of each structure. The black circular arrow indicates the rotation direction of the superstructure 
Table 1 Structural parameters of the simulated chiral overlayers as a function of the arm length of the building block, $n$

\begin{tabular}{llll}
\hline Parameter & Tripod & Cross & Active terminal segment \\
\cline { 3 - 4 } & & Homogeneous & Square $\sqrt{n^{2}+(n+1)^{2}}$ \\
\hline Unit cell shape and side, $a$ (lattice spacing) & Rhombic $\sqrt{3} \sqrt{n^{2}+n+1}$ & Square $\sqrt{1+(n+1)^{2}}$ & $\operatorname{Arctan}\left[1+\frac{1}{n}\right]$ \\
Rotation angle, $\gamma\left({ }^{\circ}\right)$ & $\operatorname{Arctan}\left[\frac{\sqrt{3}}{3} \frac{(n-1)}{(n+1)}\right]$ & $\operatorname{Arctan}(n+1)$ & Rectangular $n(n-1)$ \\
Pore shape and area, $A$ (lattice site) & Hexagonal $3 n^{2}-3 n+1$ & Square $(n-1)^{2}$ & \\
\hline
\end{tabular}

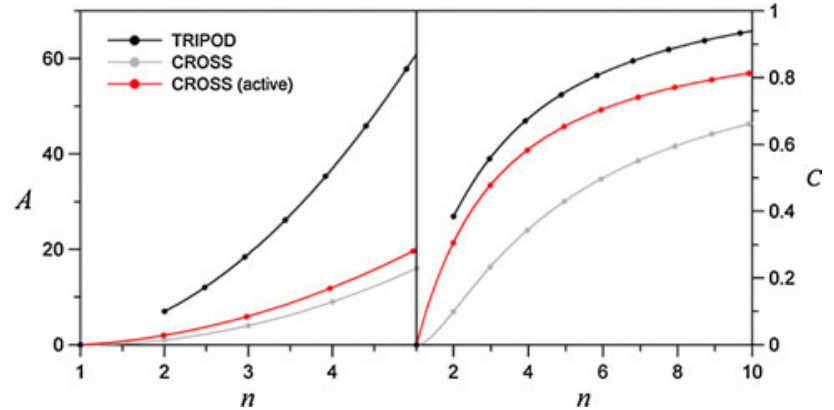

Fig. 6 Dependence of the pore area, $A$ and saturation capacity, $C$ of the simulated porous networks on the arm length of the building block, $n$. The pore area and saturation capacity are expressed in number of lattice sites and number of lattice sites per unit surface area, respectively

active terminal segments. The results shown in the right part of Fig. 6 show that the tripod-shaped and cross-shaped molecules with active terminal segments can produce ordered networks with noticeably larger total void space compared to the homogeneous tripod-shaped molecules. Moreover, the saturation capacity increases faster with $n$ for the first two types of molecules. One conclusion from the above discussion is that the elongation of the molecular arm increases noticeably the pore area of the hexagonal network while it has much less profound effect on the area of pores formed by the cross-shaped molecules. On the other hand, the saturation capacity of a network formed by the cross-shaped molecules can be noticeably increased by activating their terminal segments.

\subsection{Co-Assembly with Small Molecules}

To explore the formation of ordered structures in mixed overlayers we performed simulations in which the crossshaped molecules were co-assembled with an additive whose molecule or atom can occupy one lattice site (see Fig. 1). The simulations were carried out on a square lattice with $L=150$ using different sets of energetic parameters. The total number of adsorbed molecules and relative proportions between the components were also varied. Examples of the structures simulated for molecules with two-membered arms are shown in Fig. 7. For comparative purposes in Fig. 7a we showed large domains formed in one-component systems comprising 1500 homogeneous (left, $\varepsilon=1$ ) and heterogeneous molecules (right, $\varepsilon_{n t}=\varepsilon_{n n}=1, \varepsilon_{t t}=2$ ). Molecular arrangement within the extended domains from part A has been already shown in Figs. 3 and 5.

In the first step we examined which structure can be obtained with a mixture characterized by uniform attractive interactions. Specifically, we assumed that the cross-shaped molecules are homogeneous and that the segment-segment interaction energy is equal to the segment-additive interaction energy, that is $\varepsilon_{a n}=\varepsilon_{a t}=\varepsilon=1$. The results of the simulations performed for 1500 cross-shaped molecules mixed with 3000 molecules of the additive are shown in panel b. As it can be seen in that panel, the components assembly into ordered domains of the same symmetry as that of the domains formed by pure homogeneous molecules (see Fig. 3). In this case, however, the pores are filled by the additive, as it is shown in the magnified fragment of the superstructure. As it can be also seen, the pore filling is incomplete despite an excessive amount of the additive was used in the simulation. On the other hand, the additive molecules which are in excess stabilize smaller clusters by adsorbing on their periphery. This leads to the formation of a relatively disordered collection of small domains.

To improve the extent of ordering in the system we increased the energy of interaction between a terminal segment and an additive molecule. By doing that, the terminal segments were activated to interact stronger with the additive and to mimic the formation of donor-acceptor bonds. The results of the corresponding simulations performed for a mixture of $1500+1500$ molecules, for $\varepsilon_{a n}=$ $\varepsilon=1$ and $\varepsilon_{a t}=2$ are shown in part d of Fig. 7. As it can be seen in the figure, the increased energy of interaction between a terminal segment and an additive molecule, combined with the lower amount of the additive results in the creation of extended unichiral domains. Moreover, pores of these domains are completely filled with additive molecules. Note also that, the self-assembled structure comprises mirror-image domains which are rotated by $\pm 71.6^{\circ}$ relative to the horizontal reference axis (see Fig. 3), as calculated using Table 1. Another effect related 
Fig. 7 Snapshots of the adsorbed structures formed by the cross-shaped molecules with $n=2$ on a 150 by 150 square lattice. a Assemblies formed in one-component systems comprising 1500 homogeneous (left) and 1500 heterogeneous molecules (right). b Mixed overlayer formed by 1500 crossshaped homogeneous molecules and 3000 molecules of the additive, $\varepsilon_{a n}=\varepsilon_{a t}=\varepsilon=1$. c Mixed overlayer formed by 1000 cross-shaped homogeneous molecules and 2000 molecules of the additive, $\varepsilon_{a t}=2$, remaining energies are equal to 0 . d Mixed overlayer formed by 1500 cross-shaped homogeneous molecules and 1500 molecules of the additive, $\varepsilon_{a t}=2$, remaining energies are equal to 1 . The white arrows indicate the opposite directions of the mirror-image domains
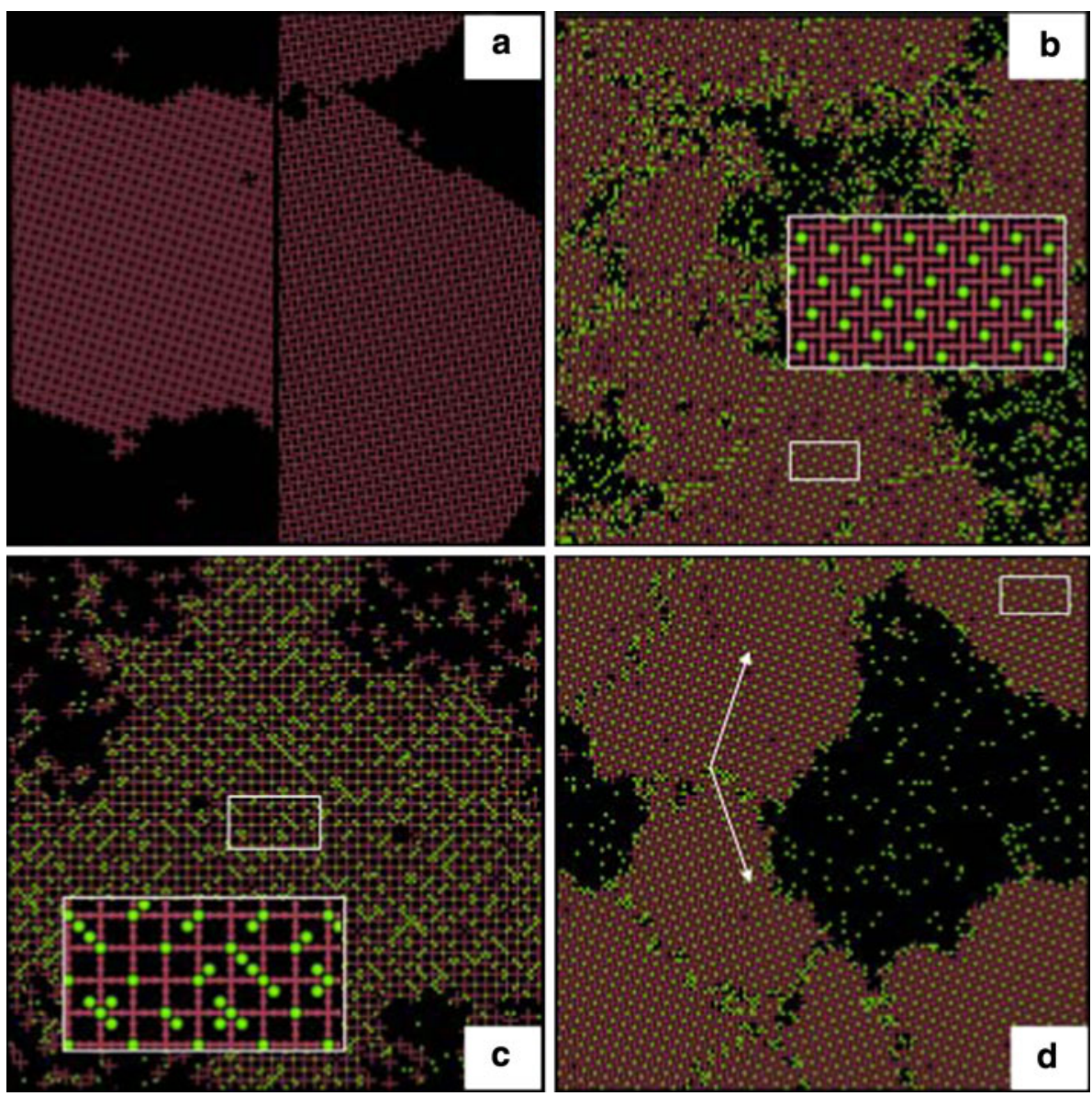

to the presence of the additive in the system is the observed coexistence of mirror-image domains. In the absence of the additive these domains would normally convert into a large unichiral superstructure for the reason which has been already discussed. Thus, the additive in our model stabilizes the near-racemic composition of the adsorbed phase and makes it observable during the simulations.

A different surface pattern emerges when the role of the terminal segment in the additive-molecule interaction becomes dominant. This is shown in panel $\mathrm{c}$ which presents the network formed by 1000 non-interacting cross-shaped homogeneous molecules co-adsorbed with 2000 molecules of the additive. This extreme case corresponds to $\varepsilon=0$ and $\varepsilon_{a t}=2$, and it can reflect, for example, the formation of strong coordination bonds between the additive and the terminal segments. In this case we can observe that the cross-shaped molecules organize into a square grid which matches the symmetry of the underlying lattice. Here, the additive plays the role of a linker, which connects four cross-shaped molecules. Note that, the resulting network is achiral and it contains pores which can accommodate up to four molecules of the additive. The magnified fragment in panel $\mathrm{c}$ shows the square pores with excess molecules of the additive adsorbed inside.
The results of this part show that the porous networks formed by the cross-shaped molecules can be used as matrices for the spatially-controlled deposition of small molecules on surfaces. As we demonstrated, the resulting co-assembled superstructures can be either chiral or achiral, depending on the nature of intermolecular interactions in the system. The symmetry of a network built of crossshaped molecules can be controlled by tuning chemical composition of this achiral building block. Of a special importance are the chiral self-assembled networks which can be further used as, for example, enantioselective adsorbents and catalysts. In the next section we present a theoretical concept on how the co-assembled superstructures obtained here can create environment for the separation of enantiomers from racemates.

\subsection{Enantioselective Surfaces from Chiral Self-Assembly}

A closer inspection of the homochiral domains presented in panels $b$ and $d$ of Fig. 7 shows that those molecular assemblies can serve as a selective adsorbent capable of differentiation between enantiomers. To demonstrate this 
Fig. 8 Mapping of the mixed ordered overlayer from Fig. 7d onto a square lattice with active (black) and inert sites (white). The left panel shows examples of adsorbed configurations of the enantiomers of a model chiral molecule comprising four segments, each occupying one lattice site. The maximum number of active sites occupied by $\mathrm{S}$ and $\mathrm{R}$ is equal 2 and 1 , respectively, as shown in the right part
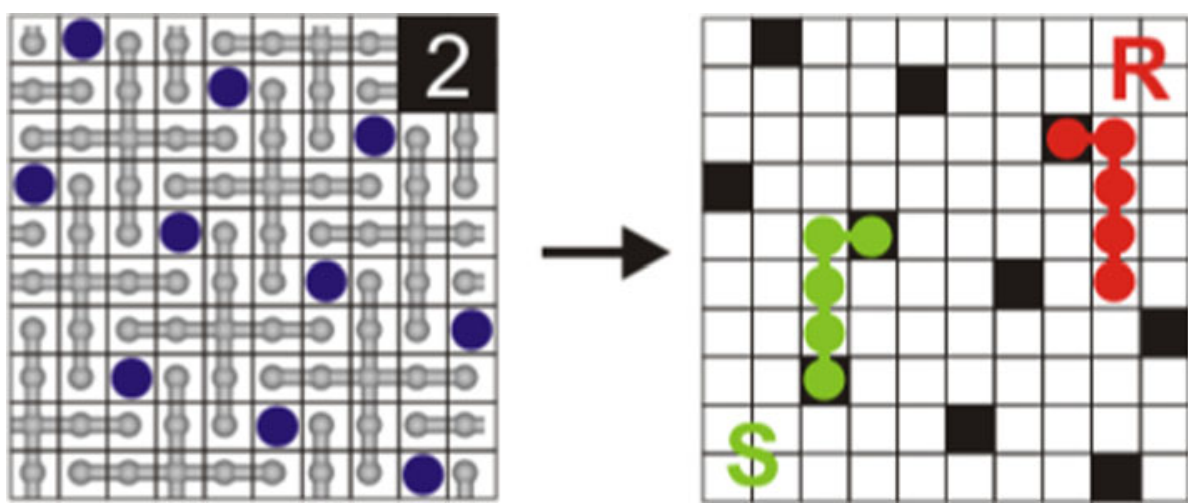

let us now use the following mapping of the mixed homochiral domain onto a square lattice:

The right part of Fig. 8 shows a simple representation of the bi-component homochiral $(\sqrt{10} \times \sqrt{10})$ structure in which the active sites (black squares) correspond to the additive molecules embedded in a set of inactive sites (white squares). The obtained surface is one example of the enantioselective surfaces proposed and studied extensively in our previous works [31-34]. As we showed using Monte Carlo simulations, a surface of this type can absorb preferentially one enantiomer of a chiral molecule, provided the molecule leaves a proper footprint on the surface. Specifically, this condition requires that one enantiomer has a footprint which interacts with more active sites on the surface compared to the other enantiomer. One example of a molecule meeting this criterion is shown in the right part of Fig. 8, although many alternative structures can be proposed [34]. As it is seen the figure, the R-enantiomer can occupy at most one active site while the S-enantiomer can occupy at most two active sites. This difference is enough to obtain preferential adsorption of $\mathrm{S}$. To show this we performed simulations for a racemic mixture $\mathrm{R}+\mathrm{S}$ adsorbing on a square $50 \times 50$ lattice with the pattern shown in the right part of Fig. 8.

The simulations of the chiral adsorption were carried out using the conventional grand canonical Monte Carlo (GCMC) method for lattice models which was described elsewhere [31]. The molecules of $\mathrm{R}$ and $\mathrm{S}$ were assumed to be rigid and composed of five identical segments. Each molecular segment was allowed to occupy one adsorption site, as shown in Fig. 8. The energy of interaction of a single segment with an active was characterized by $\varepsilon_{a}$ while for an inactive site it was assumed to be equal to $\varepsilon_{i}$. Intermolecular interactions were neglected. The results discussed below were obtained for $\varepsilon_{a}=2$ and $\varepsilon_{i}=0$. They are averages over 50 independent runs, each consisting of $2.5 \times 10^{8}$ GCMC steps.

Figure 9 shows the individual isotherms of adsorption of $\mathrm{R}$ and $\mathrm{S}$ from the racemate $\left(\theta_{S}, \theta_{R}\right.$ top) as well as the associated selectivity $S=\theta_{S} / \theta_{R}$ as functions of dimensionless pressure of the adsorbate (bottom) defined as $\exp (\mu / k T)$ where $\mu$ is the chemical potential expressed in $k T$ units.

As it clearly follows from the figure, the enantiomer $\mathrm{S}$ is preferentially adsorbed from the racemate, regardless of pressure. However, the surface has the highest preference for $\mathrm{S}$ at pressures close to zero, as reflected in the shape of the selectivity curve from the bottom part of the figure.

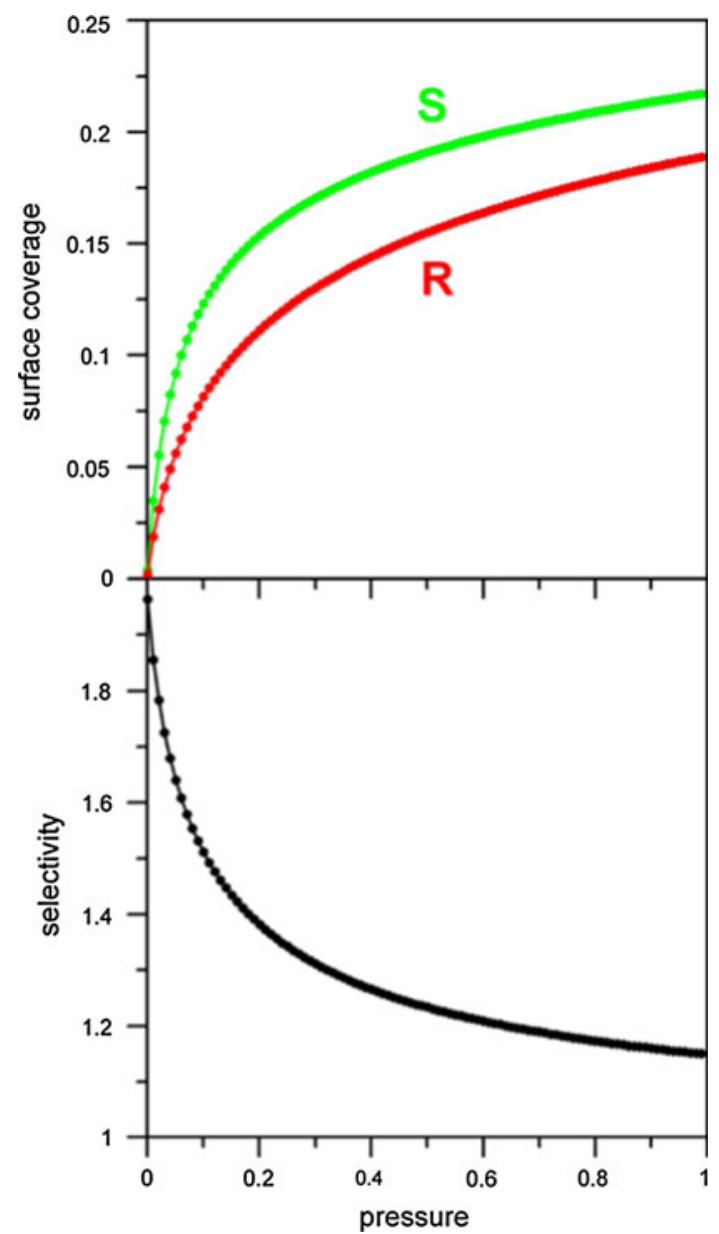

Fig. 9 Surface coverage of R and S (top) and selectivity (bottom) as functions of pressure of the racemate (arbitrary units); $\varepsilon_{a}=2, \varepsilon_{i}=0$ 
When the pressure is close to zero, the selectivity is close to 2 and it gradually drops to 1 as the pressure increases. The selectivity at the zero-pressure limit can be calculated exactly using a simple method based on counting possible configurations of $\mathrm{R}$ and $\mathrm{S}$ inside the unit cell of the surface [30-33]. This gives

$S_{0}=\lim _{p \rightarrow 0} \frac{\theta_{S}}{\theta_{R}}=\frac{x^{2}+3 x+6}{5 x+5} \quad$ where $x=e^{\varepsilon_{a}-\varepsilon_{i}}=e^{\Delta \varepsilon}$

An important feature of the selectivity at the zero-pressure limit is that it depends only on the difference between the adsorption energies $\varepsilon_{a}$ and $\varepsilon_{i}$, not on their absolute values. This provides large flexibility in tuning $S_{0}$, for example by using the additive which interacts strongly or weakly with the enantiomers. In fact, the separation can be obtained also in the second case but the preferred enantiomer would be R. This effect is shown in Fig. 10 in which we plotted the selectivity at the zero-pressure limit as a function of the energy difference $\Delta \varepsilon$.

From Fig. 10 it follows that that the selectivity grows markedly when the interaction of the additive with a segment of the adsorbate becomes stronger compared to the segment-matrix interaction. The exact value of $S_{0}$ calculated using Eq. 1 is in our case equal to 1.97 and it is marked by the red point in Fig. 10. Note that, the selectivity at the zeropressure limit is larger from 0 also when the difference $\Delta \varepsilon$ is negative. In this case, however, according to Eq. 1 the limiting value of $S_{0}$ is equal to 1.2 and it can not be further increased. For that reason, the aforementioned method of manipulating the selectivity by weakening the additivesegment interaction has only a limited applicability.

The results of this section establish a link between two theoretical concepts which involve co-assembly of achiral building blocks in two dimensions and enantioselective

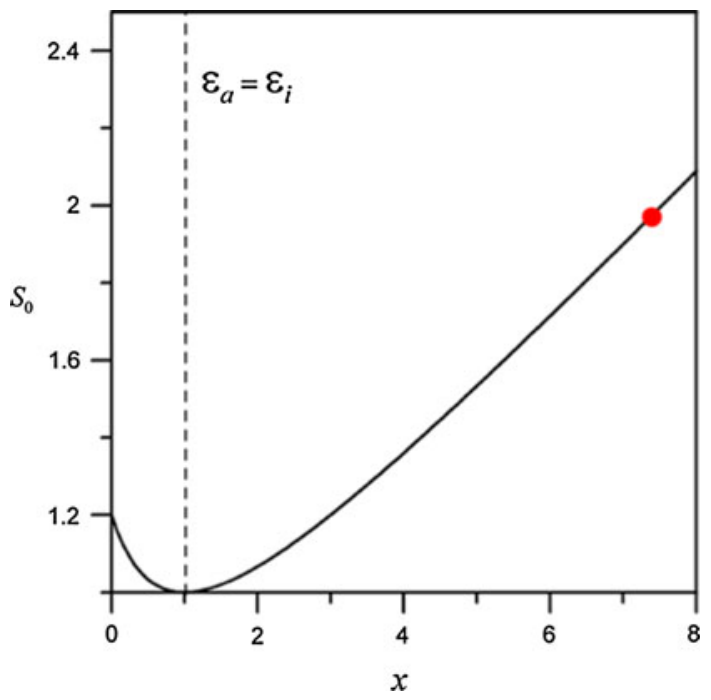

Fig. 10 Dependence of the selectivity at the zero-pressure limit on the parameter $x=\exp \left(\varepsilon_{a}-\varepsilon_{i}\right)$. The red point shows the value corresponding to the simulated system adsorption of chiral guest molecules on nanostructured surfaces. Combination of these concepts can be a useful tool for custom design of highly ordered functional matrices for adsorption and catalysis. These are the topics of our ongoing research.

\section{Conclusions}

In summary, we performed computer simulations of the selfassembly of achiral symmetric molecules in two dimensions which demonstrated that these building blocks can be effectively used to create chiral overlayers. The obtained porous networks formed by cross- and tripod-shaped molecules with longer arms $(n>1)$ comprise cavities whose size and shape can be precisely tuned by manipulating the arm length and chemical composition the molecules. Our simulations showed that these porous superstructures can serve as matrices for spatially-controlled deposition of small molecules or atoms. In this case, depending on the nature of inter-component interactions the co-assembled additive can fill the pores or it can induce the formation of a network with new symmetry. The first alternative was found to by particularly promising for the application of the co-assembled networks to the adsorptive separation of chiral guest molecules. As we showed using the GCMC method, these networks can preferentially adsorb an enantiomer whose molecular footprint is maximally compatible with the pattern formed by the incorporated additive molecules. Moreover, it was demonstrated that the selectivity of the surface can be largely increased by a suitable choice of the additive.

The theoretical models proposed in this work can be potentially used for preliminary screening of molecular libraries to select a cross- or tripod-shaped molecule which can form a network with desired structural properties. Of course, the approach adopted in this study is largely simplified and it does not account for factors such as molecular flexibility, surface heterogeneity and the formation of incommensurate adsorbed structures. Nevertheless, we believe that the results of this study can be helpful in custom designing new building blocks, as they can direct planning of their chemical synthesis.

Open Access This article is distributed under the terms of the Creative Commons Attribution Noncommercial License which permits any noncommercial use, distribution, and reproduction in any medium, provided the original author(s) and source are credited.

\section{References}

1. Sholl DS, Gellman AJ (2009) AIChE J 55:2484

2. Ahuja S (2000) Chiral separations by chromatography. Oxford University Press, Washington

3. Pirkle WH, Pochapsky TC (1989) Chem Rev 89:347 
4. Rampulla D, Gellman AJ (2004) In: Schwarz JA, Contescu CI, Putyera K (eds) Dekker encyclopedia of nanoscience and nanotechnology. Marcel Dekker, New York, pp 1113-1123

5. Zaera F (2008) J Phys Chem C 112:16196

6. Cheong WY, Huang Y, Dangaria N, Gellman AJ (2010) Langmuir 26:16412

7. Horvath JD, Koritnik A, Kamakoti P, Sholl DS, Gellman AJ (2004) J Am Chem Soc 126:14988

8. Horvath JD, Gellman AJ (2002) J Am Chem Soc 124:2384

9. Bonner WA, Kavasmaneck PR, Martin FS, Flores JJ (1974) Science 186:143

10. Hazen R, Sholl DS (2003) Nat Mater 2:367

11. Raval R (2001) CATTECH 5:12

12. Lee I, Zaera F (2005) J Phys Chem B 109:12920

13. Stacchiola D, Burkholder L, Tysoe WT (2002) J Am Chem Soc 124:8984

14. Stacchiola D, Burkholder L, Zheng T, Weinert M, Tysoe WT (2005) J Phys Chem B 109:851

15. Barth JV (2007) Annu Rev Phys Chem 58:375

16. Lin N, Stepanow S, Ruben M, Barth JV (2009) Top Curr Chem 287:1

17. Stepanow S, Lin N, Barth JV (2008) J Phys Condens Matter 20:184002

18. Kudernac T, Lei S, Elemans JJAW, De Feyter S (2009) Chem Soc Rev 38:402

19. Elemans JJAW, Lei S, De Feyter S (2009) Angew Chem Int Ed 48:7298

20. Stepanow S, Lin N, Vidal F, Landa A, Ruben M, Barth JV, Kern K (2005) Nano Lett 5:901
21. Kühne D, Klappenberger F, Decker R, Schlickum U, Brune H, Klyatskaya S, Ruben M, Barth JV (2009) J Phys Chem C $113: 17851$

22. Spillmann H, Dmitriev A, Lin N, Messina P, Barth JV, Kern K (2003) J Am Chem Soc 125:10725

23. Furukawa $\mathrm{S}$, Uji-i $\mathrm{H}$, Tahara $\mathrm{K}$, Ichikawa $\mathrm{T}$, Sonoda $\mathrm{M}$, De Schryver FC, Tobe Y, De Feyter S (2006) J Am Chem Soc 128:3502

24. Mu Z, Shu L, Fuchs H, Mayor M, Chi L (2008) J Am Chem Soc 130:10840

25. Liu B, Ran YF, Li Z, Liu S-X, Jia C, Decurtins S, Wandlowski T (2010) Chem Eur J 16:5008

26. Lu X, Hipps KW, Wang XD, Mazur U (1996) J Am Chem Soc 118:7197

27. Calmettes B, Nagarajan S, Gourdon A, Abel M, Porte L, Coratger R (2006) Angew Chem Int Ed 47:6994

28. Toader M, Gopakumar TG, Abdel-Hafiez M, Hietschold M (2010) J Phys Chem C 114:3537

29. Szabelski P, De Feyter S, Drach M, Lei S (2010) Langmuir 26:9506

30. Ha SD, Kaafarani BR, Barlow S, Marder SR, Kahn A (2007) J Phys Chem C 111:10493

31. Szabelski P, Sholl DS (2007) J Chem Phys 126:144709

32. Szabelski P (2007) Appl Surf Sci 253:5387

33. Szabelski P (2008) J Chem Phys 128:184702

34. Szabelski P (2008) Chem Eur J 27:8312 